\title{
Excitation of Discrete and Continuous Spectrum in Subdiffraction Wire-Medium Type Lenses
}

\author{
Francisco Mesa, Fellow, IEEE, Ali Forouzmand, Student Member, IEEE, \\ Alexander B. Yakovlev, Senior Member, IEEE, George W. Hanson, Fellow, IEEE, \\ Raúl Rodríguez-Berral, and Francisco Medina, Fellow, IEEE
}

\begin{abstract}
Subwavelength imaging of the near field of a magnetic line-source excitation is studied for several wire-medium (WM) lens topologies using complex-plane analysis of the radiation integral. Nonlocal homogenization is used for the wire medium, resulting in an analytical expression for the transfer function of the lens. It is shown that by evaluating the Sommerfeld integral of the transmitted field in terms of the discrete and continuous spectra provides a general framework for better understanding of electromagnetic phenomena involved with subwavelength imaging. Results are obtained for a WM slab, and for a wire medium loaded with graphene monolayers and periodic arrays of graphene patches, demonstrating the interplay of the discrete and continuous spectral components in different operating regimes of the lenses. The imaging with a stack of silver slabs is also considered for comparison purposes.
\end{abstract}

Index Terms-Graphene metamaterials, image formation, spectral decomposition, subwavelength imaging.

\section{INTRODUCTION}

$\mathbf{T}$ HE Abbe-Rayleigh diffraction limit restricts the resolution of conventional optical lenses. In order to overcome the traditional limitation on lens performance, various subwavelength near-field imaging techniques have been proposed. One well-known solution is to use a metamaterial lens exhibiting a negative refractive index. In 1968, Veselago [1] introduced the concept of a material simultaneously having negative values of permittivity $(\varepsilon)$ and permeability $(\mu)$, known

Manuscript received March 11, 2016; revised June 28, 2016; accepted September 25, 2016. Date of publication October 13, 2016; date of current version December 5, 2016. This work was supported in part by the NASA EPSCoR under Award NNX13AB31A, in part by the Spanish Ministerio de Economía y Competitividad with European Union FEDER Funds under Project TEC2013-41913-P, and in part by the Spanish Junta de Andalucía under Project P12-TIC-1435.

F. Mesa and R. Rodríguez-Berral are with the Microwaves Group, Department of Applied Physics 1, ETS de Ingeniería Informática, Universidad de Sevilla, Seville, Spain (e-mail: mesa@us.es; rrberral@us.es).

A. Forouzmand is with the Department of Electrical and Computer Engineering, Northeastern University, Boston, MA 02115 USA (e-mail: ali.forouzmand@gmail.com).

A. B. Yakovlev is with the Department of Electrical Engineering, The University of Mississippi, University, MS 38677-1848 USA (e-mail: yakovlev@olemiss.edu).

G. W. Hanson is with the Department of Electrical Engineering and Computer Science, University of Wisconsin-Milwaukee, Milwaukee, WI 53221 USA (e-mail: george@uwm.edu).

F. Medina is with the Microwaves Group, Department of Electronics and Electromagnetism, Faculty of Physics, Universidad de Sevilla, Seville 41012, Spain (e-mail: medina@us.es).

Color versions of one or more of the figures in this paper are available online at http://ieeexplore.ieee.org.

Digital Object Identifier 10.1109/TAP.2016.2617368 as a negative index material (NIM). Following this idea, Pendry [2] proposed the NIM-based perfect lens in 2000. A flat lens made from Veselago material with the relative values of both $\varepsilon$ and $\mu$ equal to -1 can effectively amplify the exponentially decaying evanescent spatial harmonics of a source field, and thus, focus both the evanescent and propagating spectra in a narrow frequency band [2]. Also, in [3] and [4], time reversal has been studied in relation to negative refraction, demonstrating subwavelength imaging for a time-reversed signal. High sensitivity to loss (the imaginary part of $\varepsilon$ and $\mu$ ) and energy dissipation lead to severe challenges in practical realization of double-negative materials [5]. Thus, the term "super lens" (rather then "perfect lens") is used for lenses made from, e.g., a plasmonic silver film [6], [7], magnetoinductive lens [8], [9], and a swiss-roll structure [10]. The physical mechanism behind the super lenses is described as an amplification of the evanescent field from the source due to excitation of coupled surface plasmon-polaritons which are supported at the interfaces of the slab. Unfortunately, such lenses are restricted to an extremely short distance between the source, lens, and image, and have a narrow resonant frequency range. However, in general, subwavelength imaging lenses have important potential biomedical and microelectronics applications in subdiffraction microscopy, lithography, tomography, sensing, and nondestructive characterization of materials [11]-[17]. As a perfect imaging device with super resolution, Maxwell's fish-eye lenses have also been proposed and studied based on non-Euclidean transformation optics in [18].

Indeed, the physical effects necessary for Pendry's subwavelength imaging take place predominately at the lens interfaces, and generally not within the metamaterial volume. Thus, it is also possible to utilize metasurfaces (quasi-2-D or farsubwavelength thin sheets), perhaps patterned, which can support surface-wave (SW) modes (surface plasmon-polaritons) within a wide range of tangential propagation wavenumbers $k_{t}>k_{0}$, instead of bulk materials [19]-[25].

Graphene, as a single atomic layer of carbon atoms with unique electronic, mechanical, and optical properties [26]-[31], has been proposed for different applications [32]-[38]. Excitement over graphene is often related to the fact that its material response can be tuned either passively by doping and various chemical/structural surface modifications, or actively by an external static electric or 
magnetic field [29]. Recently, Li and Taubner [39] presented a frequency tunable graphene lens, in which the enhancement of evanescent waves for subwavelength imaging is realized by the coupled surface plasmons supported by the graphene sheets. It should be noted that the performance of the lens sensitively depends on the distance between the graphene sheets, and the resolution reduces rapidly when the thickness of the lens is increased.

Another metamaterial lens with artificially engineered properties has been proposed in [40]-[43]. The idea is that both evanescent and propagating harmonics are transformed into transmission-line modes along the wires of a wire-medium (WM) slab, without resonant coupling and excitation of SWs of the WM which would result in the overamplification of some spatial harmonics and destruction of subwavelength imaging. It is based on the principle of canalization of the near field and does not involve the enhancement of evanescent waves [44]. The loss sensitivity of this structure is remarkably small, and the resolution is restricted only by the periodicity of the wires. It has been shown that the thickness of the wire medium should be an integer number of half wavelengths to satisfy the subwavelength imaging condition. This technique has been theoretically predicted and confirmed experimentally in [45]. It has been shown that due to the employment of metal, the properties of this lens cannot be tuned after fabrication, and the lens has narrow spectral bandwidth.

In [46], it was proposed a WM slab loaded with graphene sheets (GSs) that provides simultaneously both of the aforementioned subwavelength imaging properties of the wire medium and graphene. This lens has the potential advantages of widely tunable subwavelength imaging, and the possibility of obtaining an image even if the distance between the two graphene sheets is a significant fraction of a wavelength. In addition, the structure shows a remarkable improvement in comparison to other subwavelength imaging devices in terms of bandwidth and sensitivity to the position of the source.

It has been shown that graphene is conformable to diverse patterning schemes, resulting in interesting properties. Patterned graphene has the capability to be used in various applications such as transparent electrodes, field-effect transistors, and biosensors and energy devices. Nanoscale graphene patches (GPs), unlike a uniform graphene monolayer which is intrinsically inductive at energies below the interband transition [35], has dual capacitive/inductive properties in the low-terahertz (THz) spectrum [47], [48]. The required capacitive/inductive reactance of GPs can be obtained by a proper selection of the gap distance between patches, patch periodicity, and Fermi energy level of graphene. In [49], this unique property is exploited to design a dual-band subwavelength imaging device. A WM slab loaded with GPs can support subwavelength imaging simultaneously at two distinct tunable frequencies.

In this paper, we study subwavelength imaging in WMtype metamaterial lenses from the standpoint of the Sommerfeld excitation problem [50], where the imaging properties of the lens are accessed using a magnetic line source and considering the half-power beamwidth (HPBW) of the field at the image plane [46], [49], [51]. The radiation integral of the transmitted magnetic field is evaluated using complexplane analysis, where the original Sommerfeld integration path is deformed into the steepest descent path (SDP). The discrete (due to SW poles) and continuous [due to the branch cut (BC) contribution] spectra are identified, and dynamics of their relationship is studied in detail for different types of WM lenses. The main contribution of the paper concerns an in-depth analysis of subwavelength imaging phenomena by a careful study of excitation of the discrete and continuous spectra, as traditionally has been carried out for many challenging electromagnetic problems. A decomposition of the near field into a discrete spectrum (DS) and a continuous spectrum (CS) yields a very convenient rigorous explanation of the physical processes involved with electromagnetic phenomena due to the apparent physical meaning of these terms [52], and also creates a general framework not only for WM-type metamaterial lenses but also for other interesting phenomena involved with excitation of metamaterials. In particular, the common explanation of subwavelength imaging is related to the enhancement of evanescent waves through the resonant and nonresonant (due to absorption or losses) coupling to coupled SWs at the interfaces of the lens [2], [7], [8], [19], [39], [46], [49], [51], [53], and here we find that evaluating the Sommerfeld integral of the transmitted field in terms of the DS and CS adds a more nuanced understanding of the physics of subwavelength imaging. This paper is organized as follows. In Section II, we present the Sommerfeld radiation integral for the calculation of the magnetic field at the image plane, based on a nonlocal homogenization model for the wire medium. In Section III, the complex-plane analysis of the Sommerfeld excitation problem is presented. Subwavelength imaging for different WM topologies is studied in Section IV based on the analysis of the DS and CS. Validation of our results by comparison with commercial electromagnetic simulator is also provided in this section. Conclusions are finally drawn in Section V. A time dependence of the form $e^{j \omega t}$ is assumed and suppressed.

\section{IMAGing STRUCTURE}

The basic geometry is shown in Fig. 1, consisting of a set of vertical wires (wire medium) terminated with GPs. From this core geometry the following other structures, obtainable as limiting cases, will be studied: 1) the GPs are absent, resulting in simply a wire medium and 2) the GPs merge together to form a continuous graphene surface (GS).

An infinite magnetic line source is oriented along the $y$-direction and placed at a distance $d$ from the upper interface of the structure [Fig. 1(a)]. The magnetic current density of the line source is $\mathbf{J}_{m}=I_{0} \delta(z-d) \delta(x) \hat{\mathbf{y}}$, resulting in the incident magnetic field $\mathbf{H}(x, z)=H_{y}^{\text {inc }}(x, z) \hat{\mathbf{y}}$, with

$$
H_{y}^{\text {inc }}(x, z)=-\frac{I_{0} k_{0}^{2}}{4 \omega \mu_{0}} H_{0}^{(2)}\left(k_{0} \sqrt{x^{2}+(z-d)^{2}}\right)
$$

where $H_{0}^{(2)}$ is the zero-order Hankel function of the second kind and $k_{0}$ is the wavenumber of free space. The transmitted magnetic field at a distance $d$ from the lower interface of the 


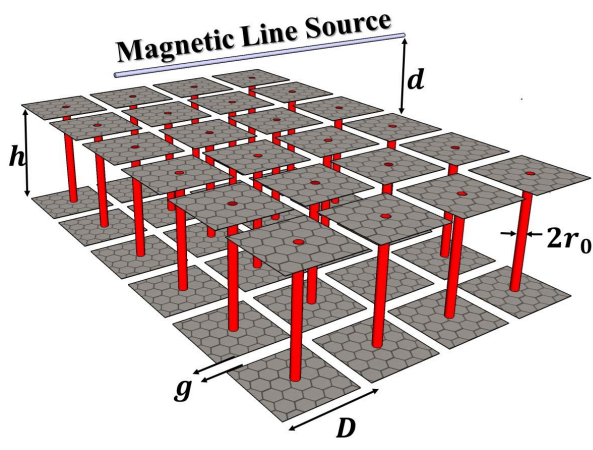

(a)

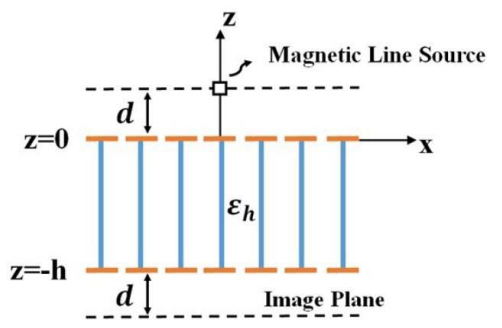

(b)

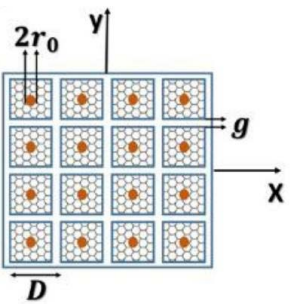

(c)
Fig. 1. (Color online) Schematics of a WM slab loaded with GPs. (a) 3-D view. (b) Cross section. (c) Top view.

structure [as shown in Fig. 1(b)] is obtained as the following Sommerfeld-type integral [51]:

$$
H_{y}(x)=\frac{I_{0} k_{0}^{2}}{j \pi \omega \mu_{0}} \int_{0}^{\infty} \frac{1}{2 \gamma_{0}} e^{-\gamma_{0}(2 d)} T\left(\omega, k_{x}\right) \cos \left(k_{x} x\right) d k_{x}
$$

where $\gamma_{0}=\left(k_{x}^{2}-k_{0}^{2}\right)^{1 / 2}$ is the vertical propagation constant in free space and $T\left(\omega, k_{x}\right)$ is the transfer function obtained with the nonlocal homogenization model for a general case of a bilayer mushroom structure loaded with graphene sheets or graphene nanostructured patch array in [46] and [49].

\section{Complex-Plane Analysis of SOMMERFELD INTEGRAL}

Given the even parity of the integrand in (2), the integration path of the above Sommerfeld integral can be extended to $C:(-\infty, \infty)$ as shown in Fig. 2(a), and closed in the lower half $k_{x}$-plane using a semicircle at $\left|k_{x}\right| \rightarrow \infty$. Using Cauchy's residue theorem, the integral is then written as a sum of residues and a BC integral [Fig. 2(a)]. Defining

$$
F\left(k_{x}\right)=\frac{I_{0} k_{0}^{2}}{j \pi \omega \mu_{0}} \frac{1}{4 \gamma_{0}} e^{-\gamma_{0}(2 d)} T\left(\omega, k_{x}\right)
$$

then

$$
\begin{aligned}
H_{y}(x)= & \int_{-\infty}^{\infty} F\left(k_{x}\right) e^{-j k_{x} x} d k_{x} \\
= & -2 \pi j \sum_{p=1}^{N_{p}} \operatorname{Res}\left\{F\left(k_{x}=k_{x, p}\right)\right\} e^{-j k_{x, p} x} \\
& +\int_{C_{b}} F\left(k_{x}\right) e^{-j k_{x} x} d k_{x}
\end{aligned}
$$

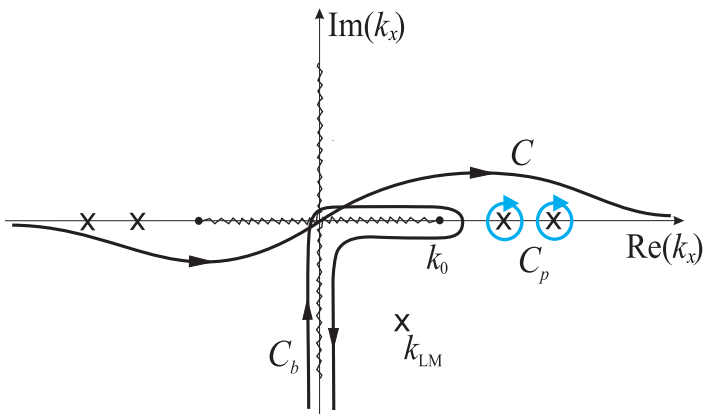

(a)

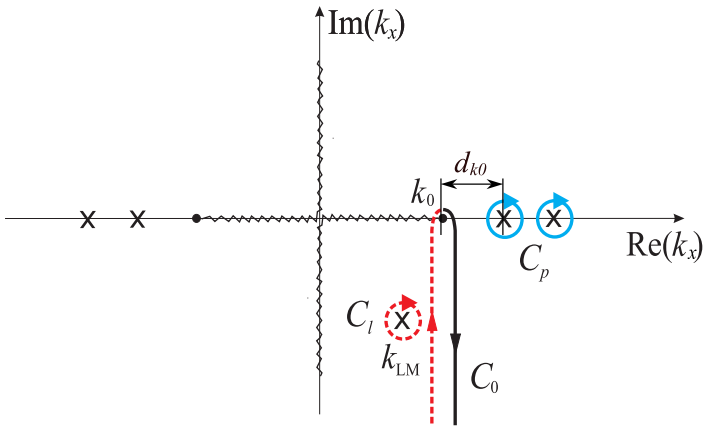

(b)

Fig. 2. (Color online) (a) Sommerfeld integration plane and deformation of the original integration path $(C)$ around the pole (cross) and branch-point (dot) singularities. (b) Deformation of the $\mathrm{BC}$ contour $C_{b}$ into the SDP $C_{0}$; leaky-wave poles (cross in red circle) may be captured.

where $N_{p}$ is the number of discrete proper poles (located on the top Riemann sheet of the Sommerfeld complex plane) of $T\left(\omega, k_{x}\right)$. The proper poles shown in Fig. 2(a) correspond to the usual case of forward bound modes (in the general lossy case they are located in the fourth quadrant). If the modes were backward, they would be located in the third quadrant (or along the negative real axis).

The integral along the path $C_{b}$ can be further deformed to $C_{0}$ formed by two vertical ascending and descending paths (ending/starting at the branch point $k_{x}=k_{0}$ ) that make up the SDP, as shown in Fig. 2(b). The ascending path lies on the bottom sheet while the descending path lies on the top sheet, such that

$$
\begin{aligned}
\int_{C_{b}} F\left(k_{x}\right) e^{-j k_{x} x} d k_{x}= & -2 \pi j \sum_{q=1}^{N_{q}} \operatorname{Res}\left\{F\left(k_{x}=k_{x, q}\right)\right\} e^{-j k_{x, q} x} \\
& +\int_{C_{0}} F\left(k_{x}\right) e^{-j k_{x} x} d k_{x}
\end{aligned}
$$

where $N_{q}$ are the improper poles of $T\left(\omega, k_{x}\right)$ captured in the deformation of $C_{b}$ to $C_{0}$ (which are commonly known as physically meaningful leaky poles) [54]. The integral along the vertical paths $C_{0}$ can be computed in the following convenient way:

$\int_{C_{0}} F\left(k_{x}\right) e^{-j k_{x} x} d k_{x}=e^{-j k_{0} x} \int_{0}^{\infty}\left[F^{+}(s)-F^{-}(s)\right] e^{-s x} d s$

where the sign \pm in $F^{ \pm}$above stands for the positive/negative value of the square root that defines $\gamma_{0}$ on the top/bottom 
Riemann sheet (in this case, the definition of the square root is taken as the standard one defined in most programming languages; namely, the real part is always positive). This latter integral is rapidly convergent because of its exponentially decaying integrand, such that (5) provides an efficient way to compute the $\mathrm{BC}$ integral in (4) provided that no physically leaky modes are captured (in the numerical examples shown below, no leaky modes are captured).

In the following sections the direct numerical integration along $C$ in Fig. 2 [equivalently, the similar path from $0 \rightarrow \infty$ in (2)] will be denoted as "Num." The DS will denote the proper residue series in (4), and the CS will denote the contribution from the $\mathrm{BC}$ integral $C_{b}$ in (5), evaluated via (6) (it is worthwhile to note here that the CS actually accounts for all the effects not directly expressible as the sum of exponentials that comprises the DS).

It is key to note here that the relevance of the DS and the CS can be anticipated by using some simple reasonings based on the value of the normalized wavenumber of the SW modes. It should be considered that the physical relevance of the DS is basically determined by the "distance," $d_{C}$, of its corresponding poles in the Sommerfeld complex plane to the original realaxis integral path $C$ in (4), as discussed in [54, Sec. III]. This rationale comes from the fact that only poles whose associated distance $d_{C}$ is small would be captured in the deformation of the original path to the SDP. Thus, for instance, $d_{C} \rightarrow 0$ for proper poles located on the real axis in Fig. 2; however, $d_{C}$ would be large for an improper pole whose real part is greater than $k_{0}$. Another important "distance," $d_{k 0}$, [in this case, Euclidean distance shown in Fig. 2(b)] to be considered is closeness of the pole to the branch point $k_{0}$, such that if the pole is very close to $k_{0}$ (in our experience, $d_{k 0} \lesssim 0.01 k_{0}$ ), then its contribution is almost completely subsumed in the branchpoint contribution. This fact leads to an enhancement of the CS rather than the DS, in similarity to the discussions provided in [52] and [53] on the effect of the proximity of pole and branch-point singularities to the saddle point in the asymptotic evaluation of diffraction integrals. In the usual case when the $\mathrm{SW}$ pole is not close to the branch point $\left(d_{k 0} \gtrsim 0.01 k_{0}\right)$ but close to the original integration path (the pole is then located on the real axis or very close to it; in our experience, $\left.d_{C} \lesssim k_{0}\right)$, the DS is expected to be significant. If the SW pole is located far from the real axis $\left(d_{C} \gtrsim k_{0}\right)$, then the DS would be almost negligible due to the high attenuation constant of its associated mode. Another interesting and common situation that appears in the present problem due to the periodic intrinsic nature of the media under analysis is the existence of SW poles on the real axis (or very close to it), but very far from the branch points $\left(\left|\operatorname{Re}\left(k_{x}\right) / k_{0}\right| \gg 1\right)$. The presence of these poles is not expected to contribute significantly to the DS since their corresponding residue is found to decrease as they are approaching the limit of the first Brillouin zone $\left(\left|\operatorname{Re}\left(k_{x}\right) / k_{0}\right| \rightarrow \lambda_{0} / 2 D\right)$.

As a summary of the above discussion, if $d_{k 0} \lesssim 0.01 k_{0}$ the "discrete" mode manifests itself as the CS, and for $d_{C} \lesssim k_{0}$ and $d_{k 0} \gtrsim 0.01 k_{0}$ it is the DS (and for $d_{C} \gtrsim k_{0}$ or $\left|\operatorname{Re}\left(k_{x}\right) / k_{0}\right| \gg 1$ it has a minimal contribution to the total field due to a negligible value of its residue). An important

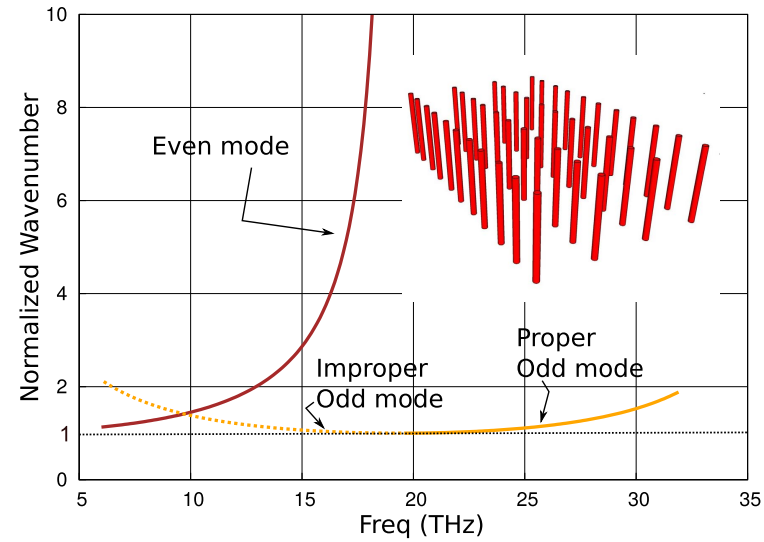

Fig. 3. (Color online) Dispersion behavior $\left(k_{x} / k_{0}\right)$ of the relevant forward even and odd SW modes for a WM slab.

conclusion of the above discussion is that there is no general simple explanation of the involved subwavelength imaging phenomena, and different possibilities arise which can be better understood on the basis of the DS + CS decomposition.

\section{Discrete And Continuous Spectrum in SUBWAVELENGTH IMAGING}

\section{A. Isolated Wire-Medium Slab}

A WM slab with period $D=215 \mathrm{~nm}$ formed by wires with radius $r_{0}=21.5 \mathrm{~nm}$ is considered (with terminating patches). The thickness of the structure is $h=7894.737 \mathrm{~nm}$ and the wires are embedded in a host medium with $\varepsilon_{h}=1$ (air). We begin with the analysis of the dispersion behavior of the relevant even and odd SW modes supported by the structure, obtained as zeros of the denominators in the expressions for the reflection coefficients $\{$ see [49, eqs. (9) and (10)]\} in the limiting case of $\sigma_{s} \rightarrow 0$. In Fig. 3, it can be seen that around $19 \mathrm{THz}$ the normalized wavenumber of the even proper bound mode increases very quickly, eventually meeting another proper mode at a slightly higher frequency to give rise to a pair of complex-conjugate modes. These complex modes with a very large attenuation constant $\left(\left|\operatorname{Im}\left(k_{x} / k_{0}\right)\right|\right)$ account for the stopband for even modes and do not play any physical role in the subwavelength imaging process; hence they are not shown in Fig. 3. It is interesting that the dispersion of the odd SW mode shows that around the frequency of the stopband for the even mode is the cutoff frequency for the odd mode. This observation is critical in the analysis to follow.

In Fig. 4, we present results for the square normalized magnitude of the magnetic field at the image plane $(d=150 \mathrm{~nm})$ calculated for different frequencies, along with the magnitude of the transfer function. Also shown in Fig. 4(a)-(e) are the individual results of the DS and CS computations, together with the total field resulting from the sum of both contributions (DS + CS). In Fig. 4(a), for validation purposes, it is also shown the direct numerical evaluation ("Num") of the original Sommerfeld integration (2). An excellent agreement is found between the time-consuming direct evaluation of (2) and the low-computationally demanding results of DS + CS. This excellent agreement is also found 


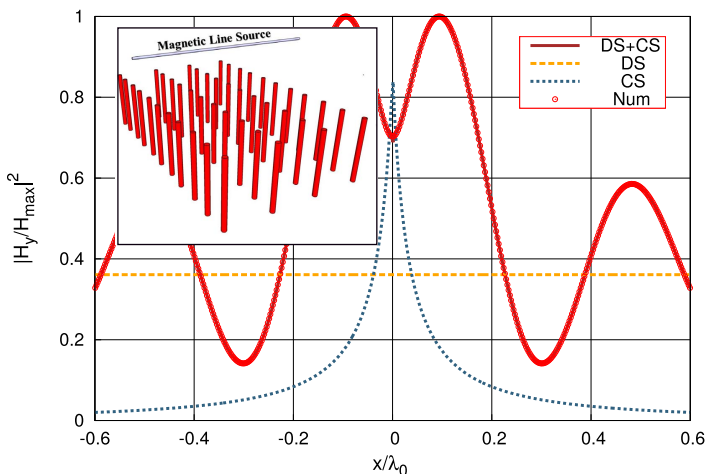

(a)

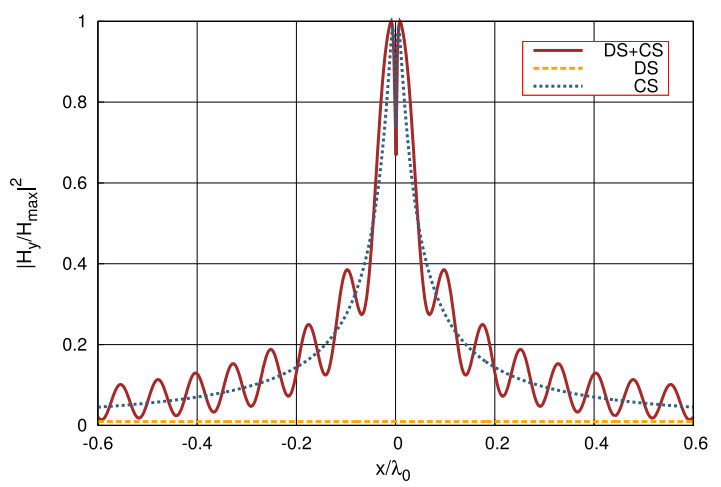

(c)

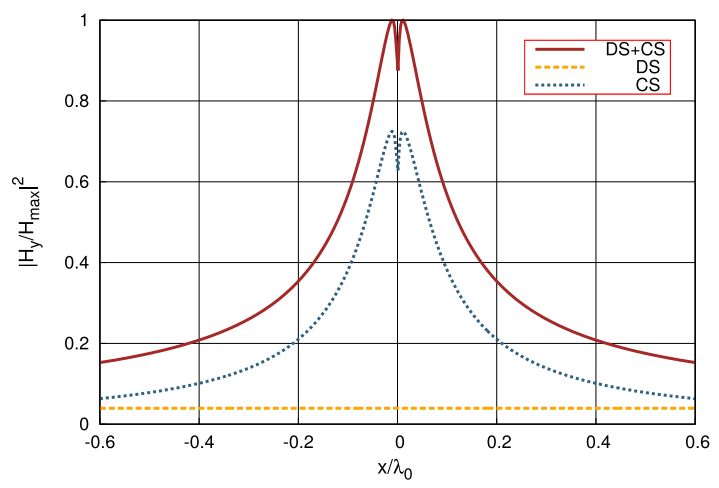

(e)

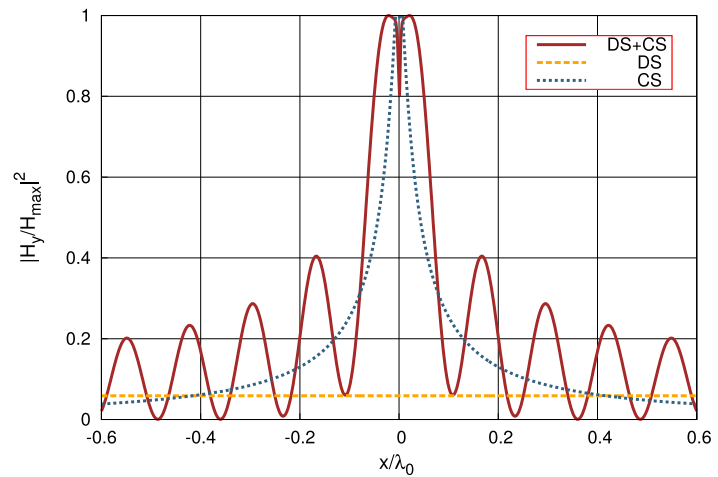

(b)

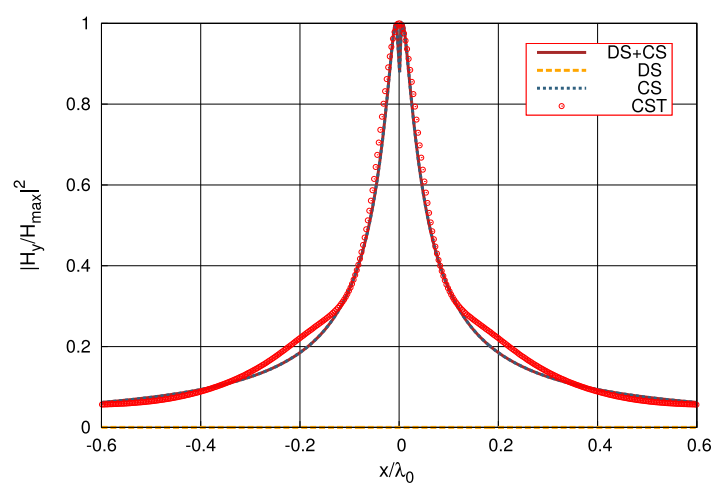

(d)

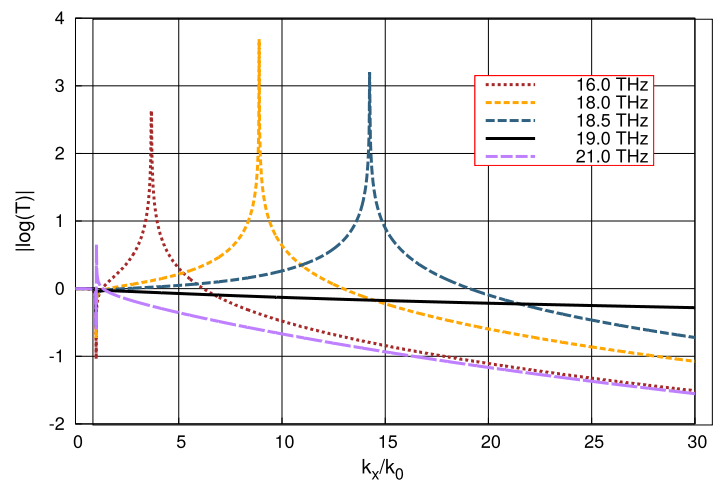

(f)

Fig. 4. (Color online) Square normalized magnitude of the magnetic field for WM slab at the image plane with $d=150$ obtained for different frequencies. (a) $16 \mathrm{THz}$. (b) $18 \mathrm{THz}$. (c) $18.5 \mathrm{THz}$. (d) $19 \mathrm{THz}$. (e) $21 \mathrm{THz}$. (f) Transfer function results (as response to evanescent waves from the source) for all of the above cases.

for all of the other examples considered in this paper, and thus it will not be explicitly shown. Additionally, as a representative example, in Fig. 4(d) it is also shown a comparison of our DS + CS field and the one obtained using the full-wave simulator CST Microwave Studio [56]. Again, the agreement of our results and CST data is quite good. This good agreement with CST results have also been found for all other cases treated in this paper, and thus this comparison will only be presented for a few representative cases.

An important first consideration is that excitation of both even and odd SW modes should be taken into account in the analysis, and the dynamics of SW poles versus frequency is important in understanding the relationship between the DS and CS contributions to the total field. It can be seen that at $16 \mathrm{THz}$ (and below; not shown in Fig. 4), the DS field is relatively significant, and constant along the image plane (in lossless structures, and assuming the existence of only one proper real SW mode, the magnitude of the DS field is constant with varying phase). This substantial DS field is caused by the excitation of the even proper forward mode with a wavenumber $k_{x} / k_{0} \approx 3.75$ (Fig. 3), as explained above. The degree to which the DS is excited becomes an important issue in subwavelength imaging, such that the total field (DS + CS) is oscillating at $16 \mathrm{THz}$ around the value corresponding to the DS field [Fig. 4(a)]. There is also an odd mode, improper real at this frequency (Fig. 3), that does not contribute to the DS field. The CS field only dominates the DS field in the vicinity of the image, and then is rapidly decaying. 
The magnitude of the transfer function at this frequency is shown in Fig. 4(f), and although its nonflat nature informs us that imaging would not be good at this frequency, it does not provide the more detailed information given by the spectrum decomposition shown in Fig. 4(a).

As we increase frequency to 18 and $18.5 \mathrm{THz}$, the DS field becomes smaller (smaller value of the residue at larger values of wavenumber $k_{x} / k_{0}$ ), and the CS field tends to approximate the total field [Fig. 4(b) and (c)]. Although the transfer function results corresponding to these two frequencies are rather similar to that corresponding to $16.0 \mathrm{THz}$ [Fig. 4(f)], it is clear that imaging at these latter frequencies is a lot better than at $16 \mathrm{THz}$, which points out that the behavior of the transfer function by itself does not provide enough information to qualitatively judge imaging ability.

At $19 \mathrm{THz}$, corresponding to the stopband for the proper bound real even mode (where $d_{C} \gg k_{0}$ ), the DS field is extremely small and the CS field captures the behavior of the total field [Fig. 4(d)]. In this case, the CS field is highly enhanced since $d_{k 0} \ll 1$ as the improper real pole of the odd mode is very close to the branch point in the $k_{x}$ complex plane (the branch point at $k_{x}=k_{0}$, associated with the vertical propagation constant $\gamma_{0}=\left(k_{x}^{2}-k_{0}^{2}\right)^{1 / 2}$, corresponds to the cutoff frequency of the odd mode). It should also be noted that this improper real pole is not captured in the deformation from $C_{b}$ to $C_{0}$ because the position of the pole in the bottom Riemann sheet is to the right of the branch point. At $19 \mathrm{THz}$ the odd mode is moving from the improper Riemann sheet to the proper Riemann sheet so that above $19 \mathrm{THz}$ it will begin to contribute to the DS. It should be noted that in the commonly used explanation of subwavelength imaging phenomena the operating frequency is typically selected very close to the frequency of the stopband for proper real bound modes, such that the density of guided modes at this frequency is extremely large which results in a resonant interaction with the near field independent of its spatial variation (see [51]). However, we should point out that the DS field corresponding to these proper bound modes is extremely small in this case (as mentioned above), and the imaging process is due to coupling to the odd proper bound mode with the pole located very close to the branch point which enhances the CS field. The results in Fig. 4(d) clearly demonstrate that the total field is the CS field at the frequency of optimum image formation (i.e., $19 \mathrm{THz}$ ), with the HPBW value of $0.112 \lambda_{0}$. This indeed is consistent with the principle of canalization with WM discussed in [57]. At this frequency the near field of the source is coupled directly to the TEM modes of the wire medium, and is canalized along the wires without resonant coupling to SW modes. However, at other frequencies shown in Fig. 4 the CS field is significant and contributes to the total field, meaning that a part of the near field is directly coupled to the TEM modes of the wire medium, despite the fact of the presence of the DS field. Fig. 4(f) reveals that at this optimum frequency, the transfer function has a flat behavior. However, despite the great differences in the transfer functions observed at 18.5 and $19.0 \mathrm{THz}$, the corresponding square normalized magnitudes of the magnetic field at the image plane are not so different, especially when comparing the values of the

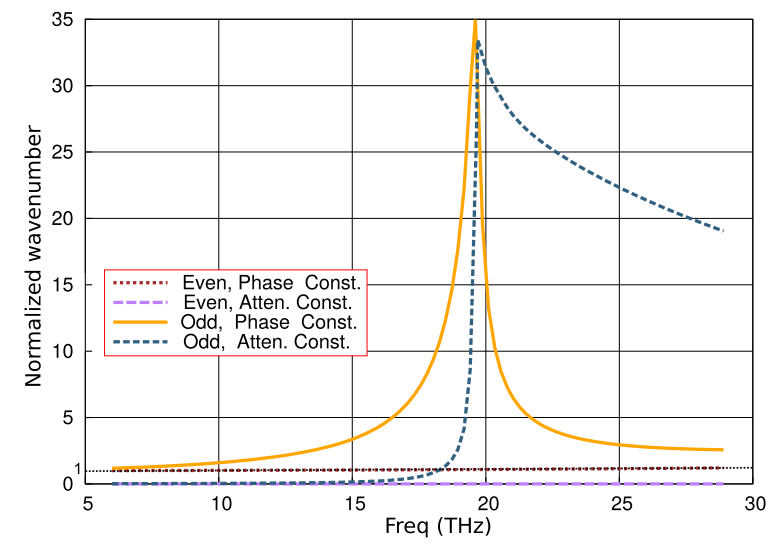

Fig. 5. (Color online) Dispersion behavior $\left(k_{x} / k_{0}\right)$ of even and odd SW modes for a WM slab terminated with continuous graphene sheets. The normalized phase constant of the even mode is very close to one in all the considered range.

square normalized magnitude above 0.4 . Another interesting observation in Fig. 4(a)-(d) is that the CS field has a very similar behavior. However, this fact is due to the normalized vertical scale employed in the figures. The absolute value of the CS is increasing from 16 to $19 \mathrm{THz}$, and then it starts to decrease again.

With an increase in frequency to $21 \mathrm{THz}$ (within the even mode stopband), there is a weak DS field and a significant CS field due to excitation of the odd proper real bound mode with $k_{x} / k_{0}=1.0084$ (very close to the branch point, which makes the DS field hardly relevant). At this frequency, there are also two complex modes: proper forward complex with $k_{x} / k_{0}=0.2863-j 26.006$ and proper backward complex with $k_{x} / k_{0}=-0.2863-j 26.006$, which do not contribute to the DS field due to a large value of the attenuation constant.

Summing up, the above discussions make it apparent that in the present case of WM the flat behavior of the transfer function is found to be related to the absence of DS (or equivalently, the full predominance of the CS) in the spectral decomposition of the total magnetic field. However, this applies to the WM case, and should not be considered as a general rule for all of the variants of structure depicted in Fig. 1, as will be discussed in the examples to follow. Also, for comparison, in the Appendix we present the results of subwavelenghth imaging with a stack of silver slabs at $900 \mathrm{THz}$ demonstrating that the DS field strongly dominates the CS field, which is consistent with the commonly used explanation related to the excitation of coupled surface-plasmon polaritons at the interfaces of the silver slabs [2], [7].

\section{B. Wire-Medium Slab Loaded With Continuous Graphene Sheets}

Here we study a WM slab terminated with continuous graphene sheets [46], with $\varepsilon_{h}=1, D=215 \mathrm{~nm}$, $r_{0}=21.5 \mathrm{~nm}$, and $h=2400 \mathrm{~nm}$. Graphene is characterized by its complex surface conductivity, which is modeled with the Kubo formula using closed-form expressions for the interband and intraband contributions [26]. In our calculations, 


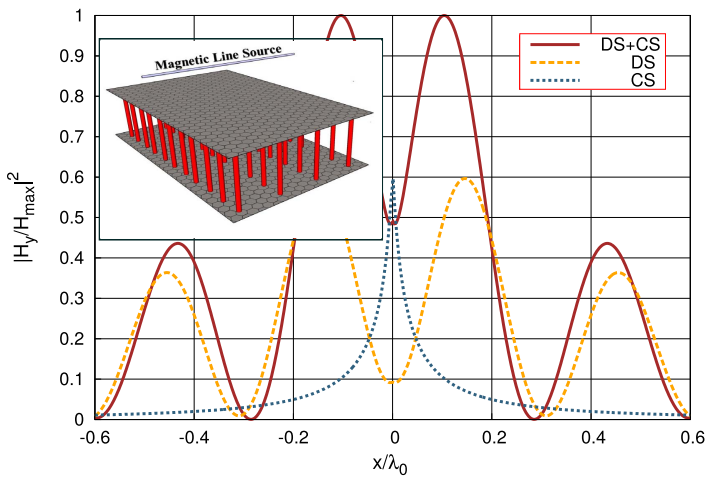

(a)

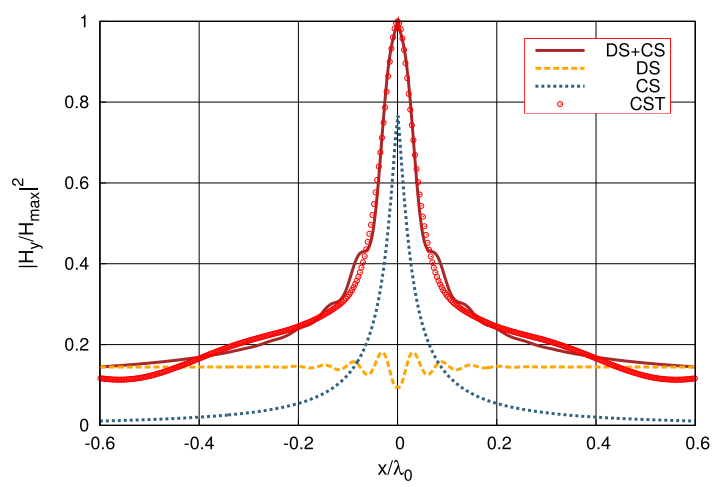

(c)

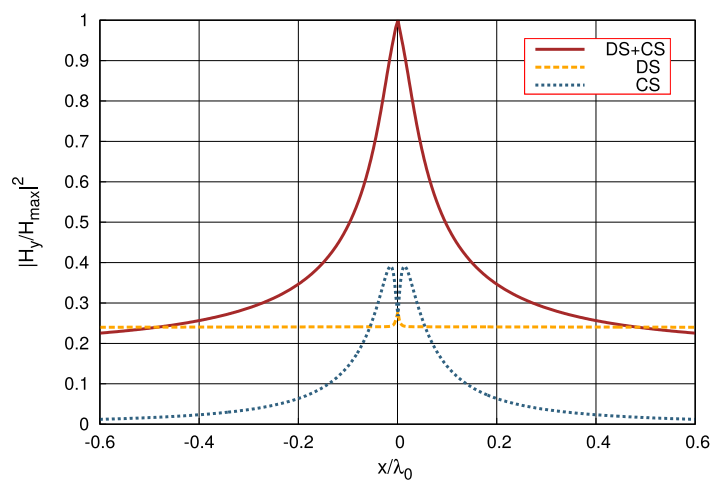

(e)

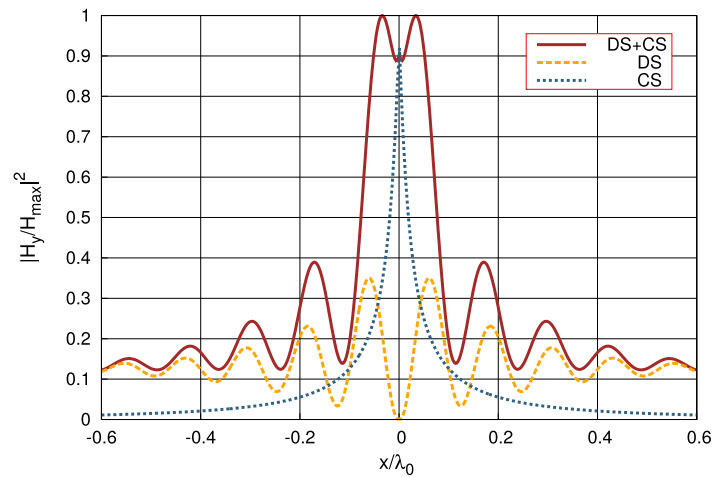

(b)

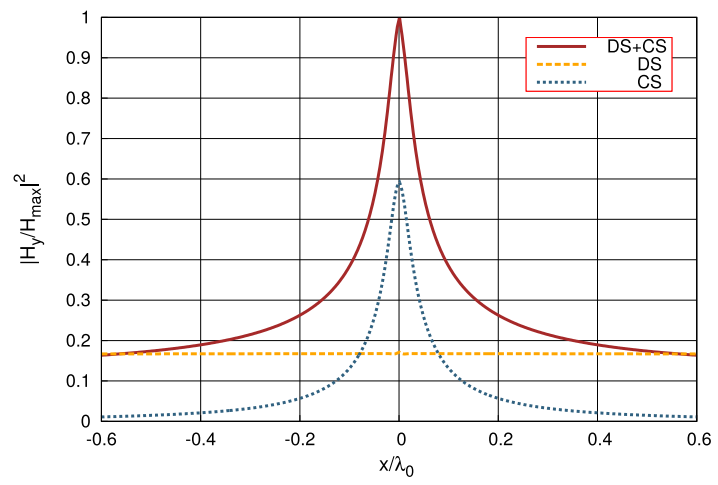

(d)

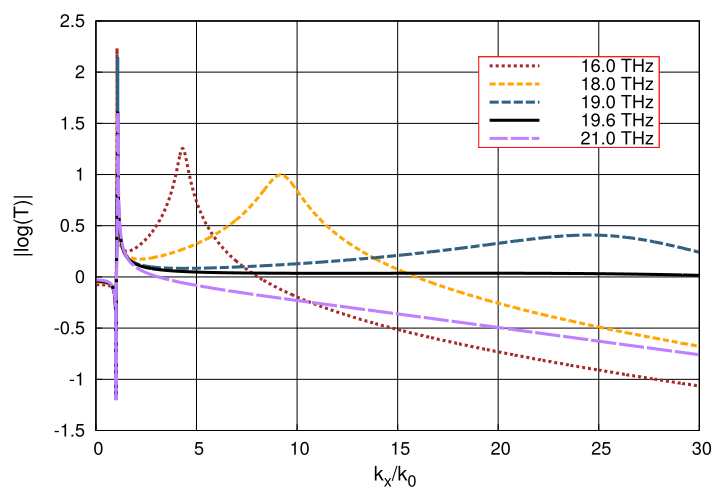

(f)

Fig. 6. (Color online) Square normalized magnitude of the magnetic field for a WM slab loaded with continuous graphene sheets at the image plane with $d=150 \mathrm{~nm}$. (a) $16 \mathrm{THz}$. (b) $18 \mathrm{THz}$. (c) $19 \mathrm{THz}$. (d) $19.6 \mathrm{THz}$. (e) $21 \mathrm{THz}$. (f) Transfer function results are shown for all of the above cases.

the following parameters for graphene have been used: the temperature, $T=300 \mathrm{~K}$, the momentum relaxation time, $\tau=0.5 \mathrm{ps}$, and the chemical potential, $\mu_{c}=1.5 \mathrm{eV}$.

The dispersion curves shown in Fig. 5 are obtained as zeros of the denominators in the expressions for the reflection coefficients $\{$ see $[49$, eqs. (9) and (10)]\} in the limiting case of $g \rightarrow 0$. The relevant modes of the structure in the considered frequency range are one even and one odd proper bound modes (both modes of forward nature). The dispersion behavior of these even and odd SW modes is shown in Fig. 5. The even SW mode has a negligible attenuation constant and a normalized phase constant $\left[\operatorname{Re}\left(k_{x} / k_{0}\right)\right]$ that ranges from 1.0072 at $6.0 \mathrm{THz}$ to 1.21 at $29.0 \mathrm{THz}$. As shown in Fig. 5, the normalized phase and attenuation constants of the odd proper bound mode increase monotonically with frequency up to around $19.6 \mathrm{THz}$, the stopband for odd modes.
At this frequency, the odd proper bound mode merges (outside the limits of the plot) with another proper complex backward mode with a large attenuation constant, which does not play any physically meaningful role in subwavelength imaging phenomena at lower frequencies. The combination of these two odd modes gives rise to a new pair of complex modes also without any relevant physical meaning due to its high attenuation constant, with only one of this pair of complex modes plotted for frequencies above $19.6 \mathrm{THz}$.

The study of the spectral decomposition of the square normalized magnitude of the magnetic field at the image plane with $d=150 \mathrm{~nm}$ is shown in Fig. 6 . At $16 \mathrm{THz}$ two proper bound modes are excited: even mode with $k_{x} / k_{0}=1.0541-$ $j 0.00047$ and odd mode with $k_{x} / k_{0}=4.3113-j 0.2231$, which is reflected in the transfer function at this frequency plotted in Fig. 6(f) (two maxima of $T\left(\omega, k_{x}\right)$ are observed 
at the pole locations). As shown in Fig. 6(a), this results in a periodic behavior of the DS field (interference of two SW modes) with a small decaying behavior caused by the small imaginary parts of the involved complex poles. Since the CS is not very relevant at this frequency, the DS field tends to approximate the total magnetic field forming two maxima in the vicinity of the image.

With the increase of frequency to $18 \mathrm{THz}$ [Fig. 6(b)] the normalized wavenumber of the odd mode increases to $k_{x} / k_{0}=9.2009-j 0.8154$, which results in a decrease of its residue. At the same time, the residue of the even-mode pole remains almost unchanged since it has slightly moved to $k_{x} / k_{0}=1.0698-j 0.00054$. The combined effect of these two even and odd modes leads to a relatively small DS field. At $19 \mathrm{THz}$ the wavenumber of the odd mode becomes very large, $k_{x} / k_{0}=18.413-j 2.812$, and with a high attenuation constant. As $d_{C} \gg k_{0}$, it means that this pole has almost no effect in the transfer function, as shown in Fig. 6(f). Since the value of the normalized wavenumber of the even mode $k_{x} / k_{0}=1.0785-j 0.00058$ has not changed very much, the magnitude of the DS field is constant since it is dominated by the residue of this even proper mode. Interestingly, the fact that phases of the DS and the CS fields are almost the same $\left(\approx k_{0} x\right)$ makes the magnitude of the DS + CS field determined by the sum of the magnitudes of the DS and the CS fields. It makes the decaying behavior of the total field to follow the one of the CS field, although the value of the total field has an offset caused by the almost constant-value DS field [Fig. 6(c)]. At this frequency, the data computed using CST Microwave Studio are also plotted, showing good agreement with our values for the total field. As we approach the frequency of the stopband for the odd proper bound mode, $19.6 \mathrm{THz}$, the only even mode is excited with $k_{x} / k_{0}=1.0841-j 0.00061$, which results in the constant value of the DS field [Fig. 6(d)], with the HPBW value of $0.1 \lambda_{0}$. At $21 \mathrm{THz}$ [Fig. 6(e)], the even mode is excited with $k_{x} / k_{0}=1.0982-j 0.00067$, which is a value that is starting to be far from the branch point and then it affects less the CS and simultaneously enhances the relative relevance of the DS. This combined effect starts to deteriorate the imaging, which gets worse as frequency increases.

By comparing the results of the WM and WM with graphene sheets it can be seen that the CS field is always present. This is due to the low-dispersion nature of the odd SW mode of the WM and the even SW mode of WM with graphene sheets, having poles located close to the branch point for a wide range of frequencies. However, unlike the WM case, the DS plays a more significant role in the presence of the graphene surface (e.g., compare Figs. 4 and 6).

\section{Wire-Medium Slab Loaded With Graphene Patches}

The WM slab loaded with GPs is analyzed with the following structural parameters: $T=300 \mathrm{~K}, \varepsilon_{h}=1, \tau=0.5 \mathrm{ps}$, $D=215 \mathrm{~nm}, g=21.5 \mathrm{~nm}, r_{0}=21.5 \mathrm{~nm}, \mu_{c}=0.5 \mathrm{eV}$, and $h=2400 \mathrm{~nm}$, with the analytical expressions of the transfer function and the surface impedance of periodic GPs given in [49].

The dispersion behavior of the even and odd physically meaningful modes of the structure at the considered frequency

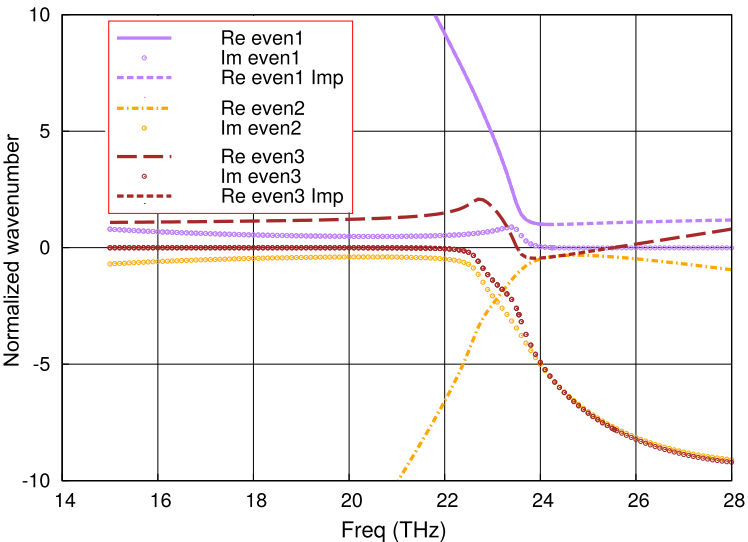

(a)

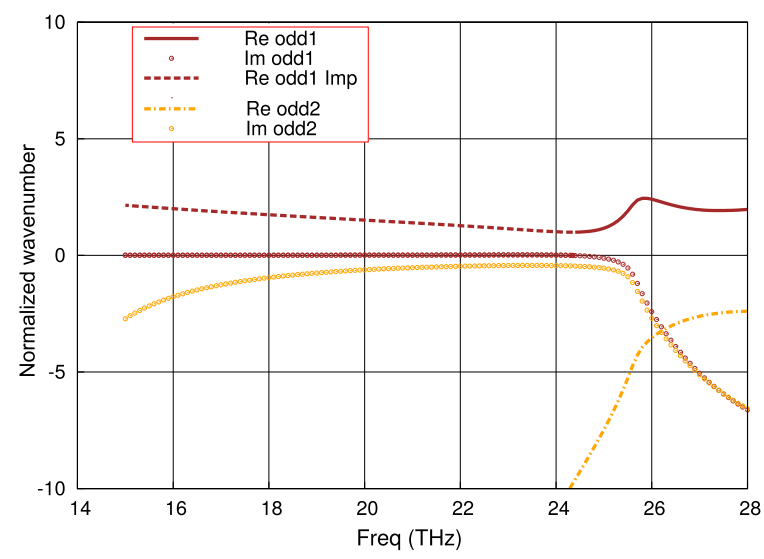

(b)

Fig. 7. (Color online) Dispersion behavior $\left(k_{x} / k_{0}\right)$ of (a) even and (b) odd modes of the WM slab loaded with GPs.

range are shown in Fig. 7(a) and (b), respectively. There are three even modes and two odd modes which are excited and contribute to the field at the image. There are two frequency regimes of subwavelength imaging with such a lens related to capacitive/inductive behavior of the GPs [47], [49].

The results for the WM slab loaded with GPs operating in the capacitive regime are shown in Fig. 8. Although not explicitly shown in this figure, numerical integration results (along the original Sommerfeld path) show again an excellent agreement with the DS + CS computation at all frequencies. At $22.5 \mathrm{THz}$ two even modes ["even2" and "even3" in Fig. 7(a)] with wavenumbers $k_{x} / k_{0}=1.8448-$ $j 0.2003$ (proper forward) and $k_{x} / k_{0}=-4.4425-j 0.7131$ (proper backward) and one odd proper backward mode ["odd2" in Fig. 7(b)] with $k_{x} / k_{0}=-16.0490-j 0.4460$ are excited. The contribution to the DS field by the odd mode is small (small value of residue due to a large real part of the normalized wavenumber). The decaying oscillatory nature of the DS field is due to the complex-valued poles of the even modes [Fig. 8(a)], which approximate well the total field. The relative small weight of the CS comes from the fact that there is not any pole close to the branch points that helps to enhance its effect. By slightly increasing the frequency to $22.65 \mathrm{THz}$ [Fig. 8(b)], the DS field rapidly reduces due to increase in the attenuation constants of the even modes: for the proper forward mode, $k_{x} / k_{0}=2.0434-j 0.4426$, and for the proper backward 


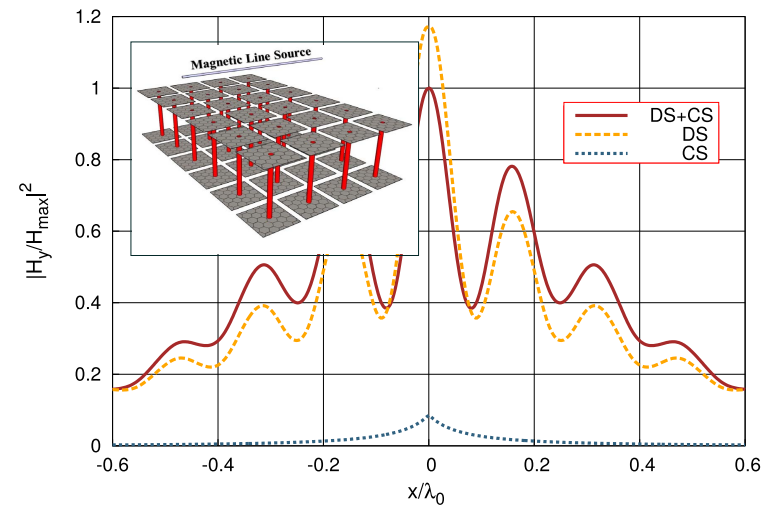

(a)

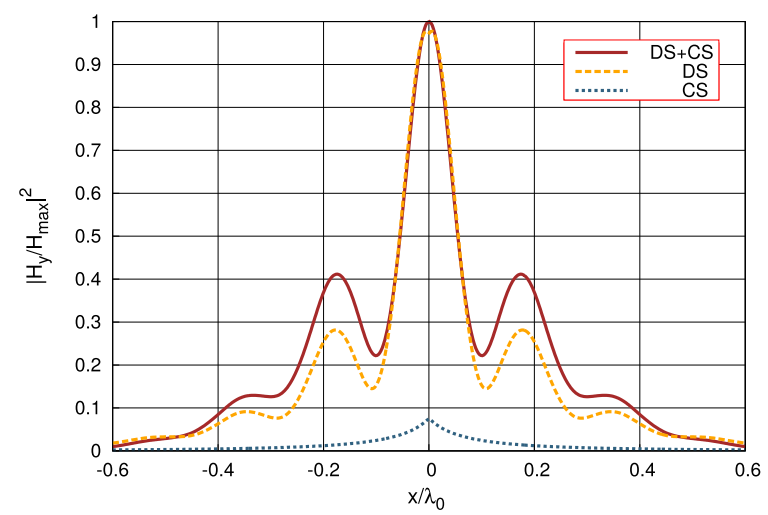

(b)

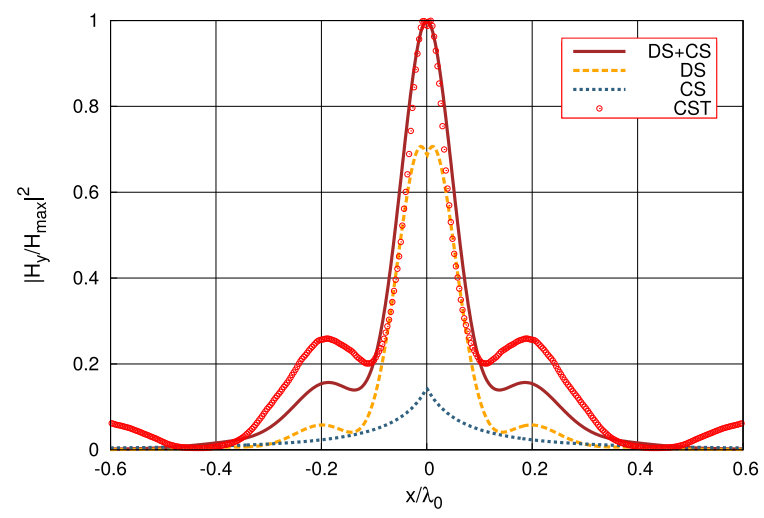

(c)

Fig. 8. (Color online) Square normalized magnitude of the magnetic field for WM slab loaded with GPs operating in the capacitive regime at the image plane with $d=150 \mathrm{~nm}$ obtained for different frequencies. (a) $22.5 \mathrm{THz}$ (b) $22.65 \mathrm{THz}$. (c) $22.8 \mathrm{THz}$.

mode, $k_{x} / k_{0}=-3.6586-j 0.9907$. At $22.8 \mathrm{THz}$, the DS field is decaying even further due to increased attenuation constants $\left(k_{x} / k_{0}=2.0544-j 0.8844\right.$, and for the proper backward mode, $\left.k_{x} / k_{0}=-3.0266-j 1.4789\right)$, resulting in better subwavelength imaging resolution, as shown in Fig. 8(c), with the HPBW value of $0.12 \lambda_{0}$. This figure also shows the data computed using CST Microwave Studio, again in good agreement with our values for the DS + CS field. In all the cases the CS field is very small in comparison to the DS field. The significance of the DS field is explained by the presence of forward and backward proper complex modes with moderate values of the real and imaginary parts of propagation constant, wherein a combined effect of these modes results in a strong

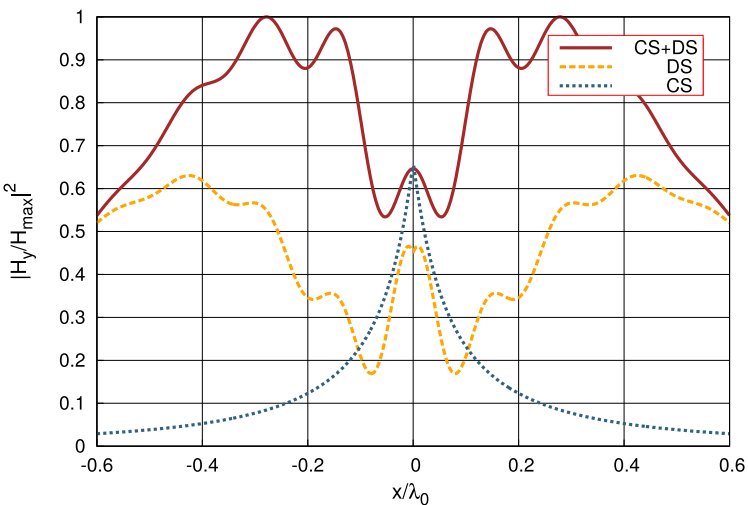

(a)

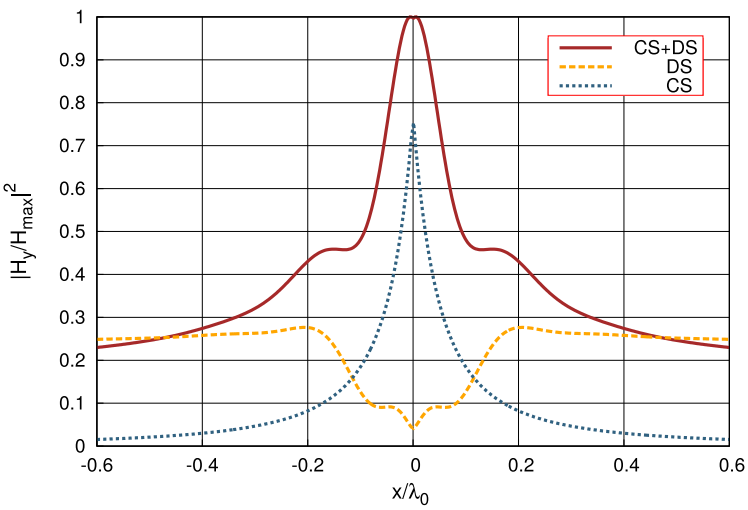

(b)

Fig. 9. (Color online) Square normalized magnitude of the magnetic field for WM slab loaded with GPs operating in the inductive regime at the image plane with $d=150 \mathrm{~nm}$ obtained for different frequencies. (a) $25.5 \mathrm{THz}$. (b) $25.9 \mathrm{THz}$

residue field which is rapidly decaying away from the source.

We should also point out here that the shape of the total field follows the concave behavior of the DS field, which dominates the CS field in the case considered above. This is in contrast to the examples of WM and WM with continuous graphene sheets where the total field follows the convex shape of the CS field, which is dominant over the DS field.

In Fig. 9, the results are for the case of a WM slab with GPs operating in the inductive regime. It is interesting to observe that the slight change in frequency gives a very different field distribution at the image. In comparison to the previous case (capacitive regime of the lens), here the CS field dominates over the DS field in the vicinity of the image, and tends to approximate the total field [at $25.9 \mathrm{THz}$ as shown in Fig. 9(b)], with the HPBW value of $0.16 \lambda_{0}$. This is due to the location of the proper pole corresponding to "even1" mode, which is now very close to the branch point. The poles corresponding to modes "even2," "odd1," and "odd2" are also considered in these computations.

\section{CONClusion}

In this paper, the complex-plane analysis of the Sommerfeld integral for a magnetic line-source over different WM topologies and its contribution to subwavelength imaging phenomena are addressed. Geometries include subwavelength imaging in an isolated WM slab, a WM slab loaded with continuous graphene sheets, and a WM slab loaded with GPs. 


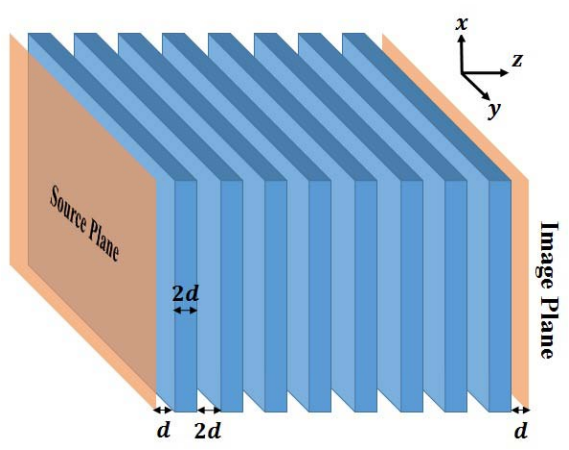

Fig. 10. (Color online) 3-D view of the stack of thin alternating silver slabs separated by air regions.

Complex-plane analysis has been performed to separate the field into discrete and continuous spectral components. This spectral decomposition has made it possible to gain a physical insight into the imaging mechanisms, and to understand that imaging can involve subtle interactions between spectral components, and is not purely associated with coupling to SWs in the vicinity of the stopband frequency for proper bound modes. In fact, we have shown that the actual contribution of highly dispersive modes at the stopband can be insignificant, and that in some cases the CS plays the most important role in imaging. In particular, for an isolated WM slab, the optimum imaging is found when the CS field greatly dominates its DS counterpart. In the WM slab loaded with continuous graphene sheets, the optimum imaging is found when the CS is still predominant but with the presence of a nonnegligible DS (however, this is not associated with highly dispersive modes at the stopband frequency, but with a low dispersive proper bound mode with the wavenumber close to the branch point). On the contrary, in the WM loaded with GPs operating in the capacitive regime, the CS is found to be almost negligible with respect to the DS when the imaging effect is optimum.

\section{APPENDIX}

Here we present a summary of the results for subwavelength imaging obtained with a stack of alternating silver slabs (with the geometry shown in Fig. 10), previously studied in [7]. The stack consists of eight silver slabs of thickness $2 d=5 \mathrm{~nm}$, separated by air regions of the same thickness. The $y$-directed line source is positioned at $d=2.5 \mathrm{~nm}$ from the first silver slab, and the image plane is at the same distance from the last silver slab in the stack. The imaging has been studied at $900 \mathrm{THz}$ with the dielectric permittivity of silver interpolated from the measured data [58].

Following the formalism presented in Sections II and III, with the transfer function of the structure obtained by the transfer matrix approach [59], the magnetic field has been obtained at the image plane with the square normalized magnitude shown in Fig. 11. It can be seen the DS field, calculated from the contributions of a proper bound forward SW with $k_{x} / k_{0}=1.2316-j 0.2264$ plus a backward SW with $k_{x} / k_{0}=-1.206-j 3.807$, strongly dominates the CS field and well approximates the total field in the vicinity of the image. This is somewhat consistent with the explanation of subwavelength imaging provided in [2] and [7] in regard

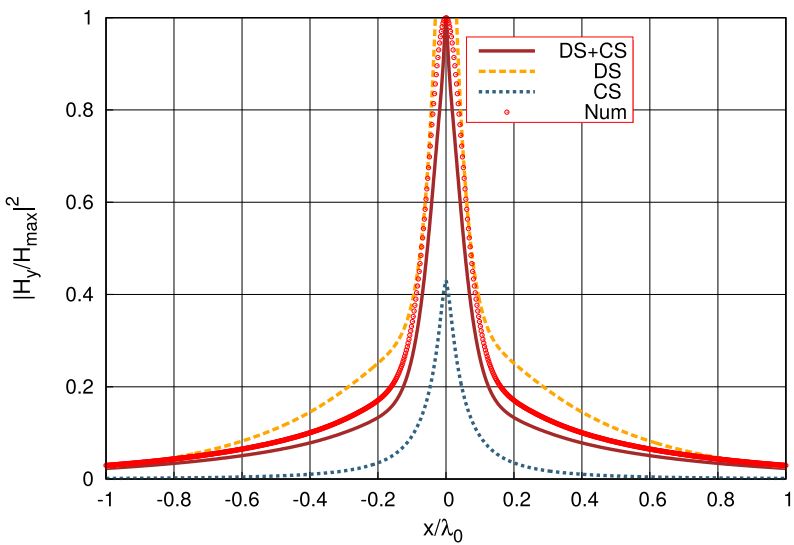

Fig. 11. (Color online) Square normalized magnitude of the magnetic field for a stack of eight silver slabs at $900 \mathrm{THz}$.

to the excitation of coupled surface-plasmon polaritons at the interfaces of the silver slabs. Comparing with the canalization regime of the wire medium slab discussed in Section IV at $19 \mathrm{THz}$, where the DS field is negligibly small and the most contribution to the total field at the image is from the CS field [Fig. 4(d)], it can be concluded that the canalization of the near field of the source with the stack of silver slabs at $900 \mathrm{THz}$ (with the relative permittivity of silver as $-0.7029-j 0.2864$ ) is of a different physical nature as it can be seen from Fig. 11.

\section{REFERENCES}

[1] V. G. Veselago, "The electrodynamics of substances with simultaneously negative values $\epsilon$ of and $\mu$," Sov. Phys.-Usp., vol. 10, no. 4, pp. 509-514, Jan. 1968.

[2] J. B. Pendry, "Negative refraction makes a perfect lens," Phys. Rev. Lett., vol. 85 , p. 3966, Oct. 2000.

[3] J. B. Pendry, "Time reversal and negative refraction," Science, vol. 322, no. 5898, pp. 71-73, Oct. 2008.

[4] G. Lerosey, J. de Rosny, A. Tourin, and M. Fink, "Focusing beyond the diffraction limit with far-field time reversal," Science, vol. 315, no. 5815, pp. 1120-1122, Feb. 2007.

[5] X. Zhang and Z. Liu, "Superlenses to overcome the diffraction limit," Nature Mater., vol. 7, no. 6, pp. 435-441, 2008.

[6] N. Fang, H. Lee, C. Sun, and X. Zhang, "Sub-diffraction-limited optical imaging with a silver superlens," Science, vol. 308, pp. 534-537, Apr. 2005.

[7] S. A. Ramakrishna, J. B. Pendry, M. C. K. Wiltshire, and W. J. Stewart, "Imaging the near field," J. Mod. Opt., vol. 50, no. 9, pp. 1419-1430, Mar. 2003.

[8] F. Mesa, M. J. Freire, R. Marquês, and J. D. Baena, "Three-dimensional superresolution in metamaterial slab lenses: Experiment and theory," Phys. Rev. B, vol. 72, pp. 235117-1-235117-6, Dec. 2005.

[9] M. J. Freire and R. Marqués, "Planar magnetoinductive lens for three-dimensional subwavelength imaging," Appl. Phys. Lett., vol. 86, pp. 182505-1-182505-3, Apr. 2005.

[10] M. C. K. Wiltshire, "Radio frequency (RF) metamaterials," Phys. Status Solidi B, vol. 244, no. 4, pp. 1227-1236, Apr. 2007.

[11] A. Grbic and G. V. Eleftheriades, "Overcoming the diffraction limit with a planar left-handed transmission-line lens," Phys. Rev. Lett., vol. 92, pp. 117403-1-117403-4, Mar. 2004.

[12] T. Taubner, D. Korobkin, Y. Urzhumov, G. Shvets, and R. Hillenbrand, "Near-field microscopy through a SiC superlens," Science, vol. 313, p. 1595 , Sep. 2006.

[13] R. Merlin, "Radiationless electromagnetic interference: Evanescent-field lenses and perfect focusing," Science, vol. 317, pp. 927-929, Aug. 2007.

[14] V. F. Fusco, N. B. Buchanan, and O. Malyuskin, "Active phase conjugating lens with sub-wavelength resolution capability," IEEE Trans. Antennas Propag., vol. 58, no. 3, pp. 798-808, Mar. 2009.

[15] S. C. Kehr et al., "Microspectroscopy on perovskite-based superlenses [invited]," Opt. Mater. Exp., vol. 1, no. 5, pp. 1051-1060, 2011. 
[16] P. Li and T. Taubner, "Multi-wavelength superlensing with layered phonon-resonant dielectrics," Opt. Exp., vol. 20, no. 11, pp. 11787-11795, 2012.

[17] H. Liu et al., "High aspect subdiffraction-limit photolithography via a silver superlens," Nano Lett., vol. 12, no. 3, pp. 1549-1554, 2012.

[18] U. Leonhardt, "Perfect imaging without negative refraction," New J. Phys., vol. 11, pp. 093040-1-093040-13, Sep. 2009.

[19] S. Maslovski, S. Tretyakov, and P. Alitalo, "Near-field enhancement and imaging in double planar polariton-resonant structures," J. Appl. Phys. vol. 96, pp. 1293-1300, Aug. 2004.

[20] S. Maslovski and S. Tretyakov, "Phase conjugation and perfect lensing," J. Appl. Phys., vol. 94, pp. 4241-4243, Oct. 2003.

[21] S. Maslovski, "Subwavelength imaging with arrays of plasmonic scatterers," Opt. Commun., vol. 285, no. 16, pp. 3363-3367, Jul. 2012.

[22] S. Maslovski and S. Tretyakov, "Perfect lensing with phase-conjugating surfaces: Toward practical realization," New J. Phys., vol. 14 pp. 035007-1-035007-22, Mar. 2012.

[23] L. Markley and G. V. Eleftheriades, "Two-dimensional subwavelengthfocused imaging using a near-field end-fire antenna-array probe," IEEE Antennas Wireless Propag. Lett., vol. 8, pp. 1025-1028, 2009.

[24] M. Karabiyik, C. Al-Amin, and N. Pala, "Deep sub-wavelength multimode tunable in-plane plasmonic lenses operating at terahertz frequencies," IEEE Trans. THz Sci. Technol., vol. 3, no. 5, pp. 550-557, Sep. 2013.

[25] O. Malyuskin and V. Fusco, "Near field enhancement and subwavelength imaging using resonantly loaded apertures," IEEE Trans. Antennas Propag., vol. 62, no. 6, pp. 3130-3140, Jun. 2014.

[26] G. W. Hanson, "Quasi-transverse electromagnetic modes supported by a graphene parallel-plate waveguide," J. Appl. Phys., vol. 104, pp. 084314-1-084314-5, Oct. 2008.

[27] B. Wang, X. Zhang, X. Yuan, and J. Teng, "Optical coupling of surface plasmons between graphene sheets," Appl. Phys. Lett., vol. 100, no. 13, pp. 131111-1-131111-4, Mar. 2012.

[28] V. P. Gusynin, S. G. Sharapov, and J. P. Carbotte, "Magneto-optical conductivity in graphene," J. Phys., Condens. Matter, vol. 19, no. 2, pp. 026222-1-026222-25, 2007.

[29] G. W. Hanson, "Dyadic Green's functions and guided surface waves for a surface conductivity model of graphene," J. Appl. Phys., vol. 103, no. 6, pp. 064302-1-064302-8, 2008.

[30] G. W. Hanson, "Dyadic Green's functions for an anisotropic, non-local model of biased graphene," IEEE Trans. Antennas Propag., vol. 56, no. 3, pp. 747-757, Mar. 2008

[31] G. Lovat, G. W. Hanson, R. Araneo, and P. Burghignoli, "Semiclassical spatially dispersive intraband conductivity tensor and quantum capacitance of graphene," Phys. Rev. B, vol. 87, no. 7, pp. 115429-1-115429-11, Mar. 2013

[32] C. S. R. Kaipa, A. B. Yakovlev, G. W. Hanson, R. Y. Padooru, F. Medina, and F. Mesa, "Enhanced transmission with a graphenedielectric microstructure at low-terahertz frequencies," Phys. Rev. B, vol. 85, pp. 245407-1-245407-6, Jun. 2012.

[33] A. Andryieuski, A. V. Lavrinenko, and D. N. Chigrin, "Graphene hyperlens for terahertz radiation," Phys. Rev. B, vol. 86 , pp. 121108-1-121108-5, Sep. 2012.

[34] T. Zhang, L. Chen, and X. Li, "Graphene-based tunable broadband hyperlens for far-field subdiffraction imaging at mid-infrared frequencies," Opt. Exp., vol. 21, no. 18, pp. 20888-20899, 2013.

[35] P.-Y. Chen and A. Alù, "Atomically thin surface cloak using graphene monolayers," ACS Nano, vol. 5, no. 7, pp. 5855-5863, 2011.

[36] E. Carrasco and J. Perruisseau-Carrier, "Reflectarray antenna at terahertz using graphene," IEEE Antennas Wireless Propag. Lett., vol. 12, pp. 253-256, 2013.

[37] P.-Y. Chen, C. Argyropoulos, and A. Alù, "Terahertz antenna phase shifters using integrally-gated graphene transmission-lines," IEEE Trans. Antennas Propag., vol. 61, no. 4, pp. 1528-1537, Apr. 2013.

[38] S. M. Raeis-Zadeh and S. Safavi-Naeini, "Analysis of electromagnetic wave scattering by graphene flakes using the generalized multipole technique," IEEE Trans. Antennas Propag., vol. 64, no. 3, pp. 1032-1038, Mar. 2016.

[39] P. Li and T. Taubner, "Broadband subwavelength imaging using a tunable graphene-lens," ACS Nano, vol. 6, no. 11, pp. 10107-10114, 2012.

[40] P. Ikonen, P. Belov, C. Simovski, and S. Maslovski, "Experimental demonstration of subwavelength field channeling at microwave frequencies using a capacitively loaded wire medium," Phys. Rev. B, vol. 73, pp. 073102-1-073102-4, Feb. 2006.

[41] P. A. Belov and M. G. Silveirinha, "Resolution of subwavelength transmission devices formed by a wire medium," Phys. Rev. E, vol. 73, pp. 056607-1-056607-9, May 2006.
[42] M. G. Silveirinha, P. A. Belov, and C. R. Simovski, "Subwavelength imaging at infrared frequencies using an array of metallic nanorods," Phys. Rev. B, vol. 75, pp. 035108-1-035108-12, Jan. 2007.

[43] M. G. Silveirinha, P. A. Belov, and C. R. Simovski, "Ultimate limit of resolution of subwavelength imaging devices formed by metallic rods," Opt. Lett., vol. 33, no. 15, pp. 1726-1728, 2008.

[44] Y. Zhao, P. A. Belov, and Y. Hao, "Subwavelength internal imaging by means of a wire medium," J. Opt. A, Pure Appl. Opt., vol. 11, pp. 075101-1-075101-6, Apr. 2009.

[45] P. A. Belov, Y. Zhao, S. Sudhakaran, A. Alomainy, and Y. Hao, "Experimental study of the subwavelength imaging by a wire medium slab," Appl. Phys. Lett., vol. 89, pp. 262109-1-262109-6, Dec. 2006.

[46] A. Forouzmand, H. M. Bernety, and A. B. Yakovlev, "Graphene-loaded wire medium for tunable broadband subwavelength imaging," Phys. Rev. B, vol. 92, pp. 085402-1-085402-14, Aug. 2015.

[47] Y. R. Padooru, A. B. Yakovlev, C. S. R. Kaipa, G. W. Hanson, F. Medina, and F. Mesa, "Dual capacitive-inductive nature of periodic graphene patches: Transmission characteristics at low-terahertz frequencies," Phys. Rev. B, vol. 87, no. 11, pp. 115401-1-115401-10, Mar. 2013.

[48] P.-Y. Chen, J. Soric, Y. R. Padooru, H. M. Bernety, A. B. Yakovlev, and A. Alù, "Nanostructured graphene metasurface for tunable terahertz cloaking," New J. Phys., vol. 15, pp. 123029-1-123029-12, Dec. 2013.

[49] A. Forouzmand and A. B. Yakovlev, "Tunable dual-band subwavelength imaging with a wire medium slab loaded with nanostructured graphene metasurfaces," AIP Adv., vol. 5, pp. 077108-1-077108-18, Jul. 2015.

[50] A. Sommerfeld, "Über die Ausbreitung der Wellen in der drahtlosen Telegraphie," Ann. Phys., vol. 333, no. 4, pp. 665-736, 1909, [Online.] Available: https:/www.cst.com/products/cstmws

[51] C. S. R. Kaipa, A. B. Yakovlev, S. Maslovski, and M. G. Silveirinha, "Near-field imaging with a loaded wire medium," Phys. Rev. B, vol. 86, pp. 155103-1-155103-10, Oct. 2012.

[52] L. B. Felsen and N. Marcuvitz, Radiation and Scattering of Waves. Englewood Cliffs, NJ, USA: Prentice-Hall, 1973.

[53] A. Ono, J.-I. Kato, and S. Kawata, "Subwavelength optical imaging through a metallic nanorod array," Phys. Rev. Lett., vol. 95, pp. 267407-1-267407-4, Dec. 2005.

[54] F. Mesa, C. Di Nallo, and D. R. Jackson, "The theory of surface-wave and space-wave leaky-mode excitation on microstrip lines," IEEE Trans. Microw. Theory Techn., vol. 47, no. 2, pp. 207-215, Feb. 1999.

[55] W. C. Chew and J. A. Kong, "Asymptotic approximation of waves due to a dipole on a two-layer medium," Radio Sci., vol. 17, no. 3, pp. 509-513, May/Jun. 1982.

[56] CST Microwave Studio, CST GmbH, 2014.

[57] P. A. Belov, C. R. Simovski, and P. Ikonen, "Canalization of subwavelength images by electromagnetic crystals," Phys. Rev. B, vol. 71, pp. 193105-1-193105-4, May 2005.

[58] P. B. Johnson and R. W. Christy, "Optical constants of the noble metals," Phys. Rev. B, vol. 6, no. 12, pp. 4370-4379, Dec. 1972.

[59] M. Born and E. Wolf, Principles of Optics, 6th ed. Oxford, U.K.: Pergamon, 1989.

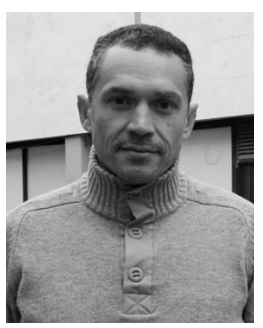

Francisco Mesa (M'93-SM'11-F'14) was born in Cádiz, Spain. He received the Licenciado and Doctor degrees in physics from the Universidad de Sevilla, Seville, Spain, in 1989 and 1991, respectively.

$\mathrm{He}$ is currently a Professor in the Departamento de Física Aplicada 1, Universidad de Sevilla. His current research interests include electromagnetic propagation/radiation in planar structures.

Dr. Mesa is currently an Associate Editor of the IEEE TRANSACTIONS ON MICROWAVE THEORY AND TECHNIQUES

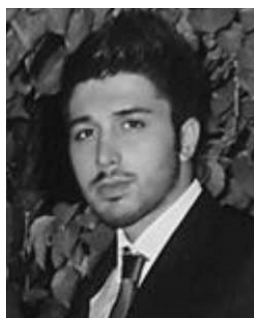

Ali Forouzmand (S'13) was born in Sabzevar, Iran, in 1990. He received the B.Sc. degree in electrical and communications engineering from the Ferdowsi University of Mashhad, Mashhad, Iran, in 2013, and the M.Sc. degree in electrical engineering (electromagnetics) from the University of Mississippi, Oxford, MS, USA, in 2015. He is currently pursuing the Ph.D. degree in electrical engineering with Northeastern University, Boston, MA, USA.

His current research interests include metamaterials, subwavelength imaging, wire media, and 


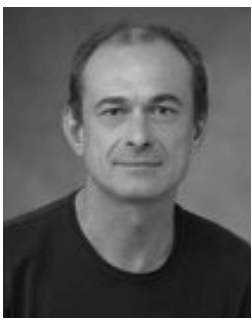

Alexander B. Yakovlev (S'94-M'97-SM'01) received the Ph.D. degree in radiophysics from the Institute of Radiophysics and Electronics, National Academy of Sciences, Kharkov, Ukraine, in 1992, and the $\mathrm{Ph} . \mathrm{D}$. degree in electrical engineering from the University of Wisconsin-Milwaukee, Milwaukee, WI, USA, in 1997.

In 2000, he joined the Department of Electrical Engineering, University of Mississippi, Oxford, MS, USA, as an Assistant Professor and became an Associate Professor in 2004. Since 2013, he has been a Full Professor of Electrical Engineering. His current research interests include mathematical methods in applied electromagnetics, homogenization theory, high-impedance surfaces for antenna applications, electromagnetic bandgap structures, metamaterial structures, wire media, graphene, cloaking, theory of leaky waves, transient fields in layered media, and catastrophe and bifurcation theories.

Dr. Yakovlev was a recipient of the Young Scientist Award at the 1992 URSI International Symposium on Electromagnetic Theory, Sydney, NSW, Australia, and the Young Scientist Award at the 1996 International Symposium on Antennas and Propagation, Chiba, Japan. In 2003, he was also a recipient of the Junior Faculty Research Award in the School of Engineering at the University of Mississippi. From 2003 to 2006, he was an Associate Editorin-Chief of the Applied Computational Electromagnetics Society Journal and from 2005 to 2008 was an Associate Editor of the IEEE TRANSACTIONS on Microwave Theory and Techniques. He is a member of the URSI Commission B. He is a Co-Author of the book Operator Theory for Electromagnetics: An Introduction (New York, NY, USA: Springer, 2002).

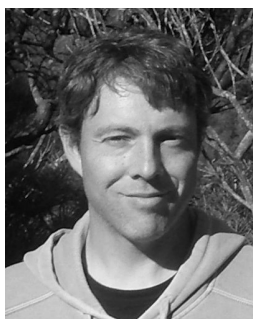

George W. Hanson (S'85-M'91-SM'98-F'09) was born in Glen Ridge, NJ, USA, in 1963. He received the B.S.E.E. degree from Lehigh University, Bethlehem, PA, USA, the M.S.E.E. degree from Southern Methodist University, Dallas, TX, USA, and the Ph.D. degree from Michigan State University, East Lansing, MI, USA, in 1986, 1988, and 1991, respectively.

From 1986 to 1988, he was a Development Engineer with General Dynamics, Fort Worth, TX, USA, where he was involved in radar simulators. From 1988 to 1991, he was a Research and Teaching Assistant at the Department of Electrical Engineering, Michigan State University. He is currently a Professor of Electrical Engineering and Computer Science with the University of Wisconsin-Milwaukee, Milwaukee, WI, USA. His current research interests include nanoelectromagnetics, plasmonics, quantum optics, and mathematical methods in electromagnetics.

Dr. Hanson is a member of the URSI Commission B, Sigma Xi, and Eta Kappa $\mathrm{Nu}$, and was an Associate Editor of the IEEE TRANSACTIONS ON ANTENNAS AND PROPAGATION from 2002 to 2007. In 2006, he received the S.A. Schelkunoff Best Paper Award from the IEEE Antennas and Propagation Society. He is a Co-Author of the book Operator Theory for Electromagnetics: An Introduction (New York, NY, USA: Springer, 2002), and the Author of Fundamentals of Nanoelectronics (New Jersey, NJ, USA: Prentice-Hall, 2007).

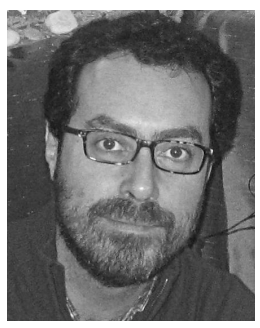

Raúl Rodríguez-Berral was born in Casariche, Seville, Spain, in 1978. He received the Licenciado (M.Sc.) and Ph.D. degrees in physics from the University of Seville, Seville, in 2001 and 2008, respectively.

In 2002, he joined the Department of Applied Physics 1, University of Seville, where he is currently an Assistant Professor. His current research interests include the study of the spectrum and the excitation of periodic and nonperiodic planar structures and high-frequency circuit modeling.

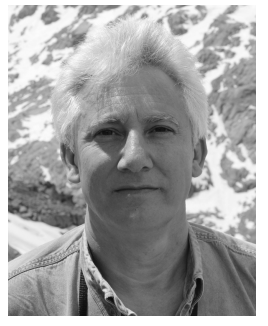

Francisco Medina (M'90-SM'01-F'10) was born in Puerto Real, Cádiz, Spain. He received the Licenciado and Doctor degrees in physics from the University of Seville, Seville, Spain, in 1983 and 1987, respectively.

$\mathrm{He}$ is currently a Professor of Electromagnetism with the Department of Electronics and Electromagnetism, University of Seville, where he was the Head of the Microwaves Group. His current research interests include analytical and numerical methods for planar structures, anisotropic materials, periodic electromagnetic structures and metamaterials, and printed passive microwave devices. He has extensively published on these topics in international journals and conferences. He acts as a Reviewer for IEEE, IET, AIP, and IOP journals and is an Associate Editor of the International Journal of Microwave and Wireless Technologies. He has also been a member of the TPCs of a number of local and major international conferences. 